\title{
Aging effects on prefrontal cortex oxygenation in a posture-cognition dual- task: an fNIRS pilot study
}

\author{
Uros Marusic ${ }^{1,2^{*}}$ D, Wolfgang Taube ${ }^{3}$, Shawnda A. Morrison ${ }^{4}$, Lea Biasutti ${ }^{5}$, Bruno Grassi ${ }^{5}$, Kevin De Pauw ${ }^{6}$, \\ Romain Meeusen ${ }^{6}$, Rado Pisot ${ }^{1,7}$ and Jan Ruffieux ${ }^{3}$
}

\begin{abstract}
Background: The aging process alters upright posture and locomotion control from an automatically processed to a more cortically controlled one. The present study investigated a postural-cognitive dual-task paradigm in young and older adults using functional Near-Infrared Spectroscopy (fNIRS).

Methods: Twenty healthy participants (10 older adults $72 \pm 3$ y, 10 young adults $23 \pm 3$ y) performed a cognitive (serial subtractions) and a postural task (tandem stance) as single-tasks (ST) and concurrently as a dual-task (DT) while the oxygenation levels of the dorsolateral prefrontal cortex (DLPFC) were measured.

Results: In the cognitive task, young adults performed better than older adults in both conditions (ST and DT) and could further increase the number of correct answers from ST to DT (all $p s \leq 0.027$ ) while no change was found for older adults. No significant effects were found for the postural performance. Cerebral oxygenation values $\left(\mathrm{O}_{2} \mathrm{Hb}\right)$ increased significantly from baseline to the postural ST $(p=0.033)$, and from baseline to the DT $(p=0.031)$ whereas no changes were found in deoxygenated hemoglobin ( $\mathrm{HHb}$ ). Finally, the perceived exertion differed between all conditions ( $p \leq 0.003)$ except for the postural ST and the DT $(p=0.204)$.

Conclusions: There was a general lack of age-related changes except the better cognitive performance under motor-cognitive conditions in young compared to older adults. However, the current results point out that DLPFC is influenced more strongly by postural than cognitive load. Future studies should assess the different modalities of cognitive as well as postural load.
\end{abstract}

Keywords: Postural control, Balance, Dual-tasking, Executive control, Elderly, Aging, Functional near-infrared spectroscopy (fNIRS), Attention

\section{Introduction}

Aging causes a shift from an automatic to a more cortical control of upright posture and locomotion [1-3]. Older adults show increased and more widespread involvement of cortical areas for postural control compared to young adults; notably in the prefrontal cortex [3, 4]. These over-activations in older adults have been interpreted as a dedifferentiation of brain activation, or as a compensation for age-related declines in brain structure and function

\footnotetext{
*Correspondence: uros.marusic@zrs-kp.si; umarusic@outlook.com ${ }^{1}$ Institute for Kinesiology Research, Science and Research Centre Koper, Garibaldijeva 1, Koper, Slovenia

${ }^{2}$ Department of Health Sciences, Alma Mater Europaea - ECM, Maribor, Slovenia

Full list of author information is available at the end of the article
}

[3]. This increase in cortical engagement implies that postural processing is more attention-demanding in this age group compared to their younger counterparts. Further evidence for a more conscious, attention-demanding postural control strategy in older age has been provided indirectly by studies using a posture-cognition dual-task paradigm $[5,6]$.

In everyday life, it is common to experience situations in which a postural task (e.g., standing or walking) is performed concurrently with a secondary task. Older adults are particularly prone to dual-task costs in situations where attentional resources are shared between a postural and one (or more) additional tasks. In fact, it has been shown that the costs of performing a postural and a cognitive task

(c) The Author(s). 2019 Open Access This article is distributed under the terms of the Creative Commons Attribution 4.0 International License (http://creativecommons.org/licenses/by/4.0/), which permits unrestricted use, distribution, and reproduction in any medium, provided you give appropriate credit to the original author(s) and the source, provide a link to the Creative Commons license, and indicate if changes were made. The Creative Commons Public Domain Dedication waiver (http://creativecommons.org/publicdomain/zero/1.0/) applies to the data made available in this article, unless otherwise stated. 
concurrently are greater in older adults [7-10], implying an increased risk of falls. Indeed, the reduced ability to allocate sufficient attentional resources to postural tasks may account for the high number of falls in the elderly [11]. Performance in balance and/or locomotor tasks under dual-task conditions has been shown to be a good predictor of falls $[12,13]$.

The prefrontal cortex plays a prominent role in postural, cognitive, and dual-task performance. Studies using functional near-infrared spectroscopy (fNIRS) have shown significant activation in the prefrontal cortex, including the dorsolateral prefrontal cortex (DLPFC), during walking [14, 15], in response to a perturbation to upright stance [16], or during performance of a balance task in a semi-immersive virtual reality environment $[17,18]$. In the latter two studies, prefrontal oxygenation levels were positively correlated with task difficulty. Higher prefrontal oxygenation levels during standing were found in patients with Parkinsonian syndromes compared to healthy older adults [19]. Thus, it has been suggested that the prefrontal cortex is critical for one's ability to selectively allocate (visuospatial) attention $[15,16]$ and to integrate visual and proprioceptive information [18] in order to maintain or regain postural stability. Besides this role in the processing of postural tasks, the DLPFC also plays a key role in working memory tasks [2022]. Thus, there is evidence that the prefrontal cortex, particularly DLPFC, is crucial for the performance of postural dual-tasks. Studies using dual-task walking paradigms found increases in prefrontal activity when an attention-demanding task was added to normal walking in young adults, while older adults showed smaller increases [14] or even deactivations [23].

To our knowledge, no age-comparative fNIRS study exists to date that has investigated such effects using a standing postural task in combination with a working memory task. Furthermore, in the studies cited above [14, 23], oxygenation levels were measured during walking alone, or during dual-task walking, but not during single-task performance of the additional task. Therefore, the aim of this study was to investigate the effect of performing a standing and a working memory task alone, and concurrently, on prefrontal activity in young and older adults, and to see whether differences in task performance could be explained by changes in prefrontal cortex activity. For this purpose, we measured behavioral performance as well as prefrontal oxygenation levels using fNIRS during standing, counting backwards, and when the two tasks were combined, in both young and older adults.

\section{Methods}

All procedures were carried out in accordance with the ethical standards of the 1964 Declaration of Helsinki and were approved by the National Medical Ethics Committee. Written informed consent was obtained from all participants prior to the study and no payment was provided for participation.

\section{Participants}

Older adults were identified and contacted from the clinical database of an existing EU-funded project entitled "Physical Activity and Nutrition for Quality Ageing" (PANGeA). For the purpose of this study, 10 healthy older adults (for details see Table 1) were randomly selected from a pool of 152 volunteers and recalled to the laboratory for further testing. In addition, 10 healthy young adults (for details see Table 1) were recruited and randomly selected from the database of the study program Applied Kinesiology (University of Primorska, Slovenia). Exclusion criteria included any history or current symptoms of: severe acute metabolic, neuromuscular, and cardiovascular diseases, excessive obesity (over $45 \%$ fat), infectious diseases, cancer, bleeding, physical exhaustion, mild cognitive impairment or dementia, critical ischemia of the lower limbs, and patients unable to complete all measurement protocols. All participants

Table 1 Participants' characteristics

\begin{tabular}{llll}
\hline & Young adults $(N=10)$ & Older adults $(N=10)$ & $p$ value \\
\hline Sex & 7 women & 6 women & $<0.001$ \\
Age (years) & $22.6 \pm 2.8$ & $72.3 \pm 3.2$ & $166.8 \pm 8.6$ \\
Height $(\mathrm{cm})$ & $173.4 \pm 6.6$ & $73.8 \pm 11.2$ & 0.074 \\
Weight $(\mathrm{kg})$ & $75.3 \pm 12.2$ & $14.1 \pm 2.1$ & 0.798 \\
Education duration (years) & $14.3 \pm 1.6$ & $28.0 \pm 1.2$ & \\
MoCA total score & & $3.9 \pm 0.6$ & 0.828 \\
Subjective health assessment: & & $4.0 \pm 0.8$ & 0.123 \\
a.) General health status & $4.3 \pm 0.5$ & $4.2 \pm 0.6$ & 0.631 \\
b.) Physical fitness & $4.2 \pm 0.6$ & $4.3 \pm 0.7$ & 0.353 \\
c.) Mental health & $4.5 \pm 0.5$ & 0.853 \\
d.) Quality of life & $4.4 \pm 0.5$ &
\end{tabular}

Note: Data are mean \pm SD. MoCA, Montreal Cognitive Assessment 
were right-handed and had normal or corrected-to-normal vision. All participants indicated on a 5-point Likert scale, where 1 represents a minimum (very bad) and 5 a maximum (very good) score, how much they agreed on their general health status, physical fitness, mental health, and general quality of life. After conclusion of all measurements and a short break, older adults were additionally evaluated with Montreal Cognitive Assessment (MoCA) tool. Characteristics of all participants are presented in Table 1.

\section{Study design and experimental tasks}

This between-group design study consisted of a dual-task (DT) paradigm that combined a postural task with a cognitive task. The postural task consisted in standing as still as possible in a tandem position while focusing on a black point placed at eye-level approximately one meter in front of the participants. A force plate (AMTI HE600600-2 k, Advanced Mechanical Technology, Inc., Watertown, MA, USA) was used to measure displacements of the center of pressure (COP) in both medio-lateral $(\mathrm{m}-\mathrm{l})$ and antero-posterior (a-p) directions. All postural parameters (mean sway path, mean frequency, and mean amplitude) were obtained from the Wise-Coach software (Wise Technologies, Ljubljana, Slovenia). Furthermore, the cognitive task consisted in subtracting serial threes from a randomly chosen number between 400 and 500. Participants were instructed to perform as many correct subtractions as possible while prioritizing correctness over speed and the number of correct answers was counted for each trial.

Upon arrival, all participants received a familiarization period (approximately $10 \mathrm{~min}$ ), in which they got used to the measuring environment and equipment, as well as the postural and cognitive tasks. Afterwards, the following conditions were performed, during which prefrontal cortex oxygenation was measured: 1) a baseline reference condition (for the fNIRS analysis) that consisted in quiet standing, 2) cognitive single-task (Cognitive ST), 3) postural single-task (Tandem ST), and 4) the cognitive and postural tasks concurrently, i.e., dual-task (DT). The order of the latter two conditions was randomized between participants. Two trials of $60 \mathrm{~s}$ and were performed in each condition. All measurements were carried out in a separate and quiet room to avoid any external disturbances. At the end of each trial, participants were asked to subjectively rate their perceived exertion (0-10 Borg scale). Finally, dual-task effects (DTEs) were calculated for all COP parameters and for the cognitive performance following the formula published in [24] and cognitive-motor dual-task interference [25] was further added.

\section{Cerebrovascular setup}

Measurement of prefrontal cortex oxygenation was performed using a single-distance continuous wave fNIRS set-up (Oxymon, Artinis, The Netherlands), using methods described elsewhere [26]. Briefly, this device contained a headset that held a near-infrared emitter with laser light at 780 and $850 \mathrm{~nm}$ and a detector pair placed above the DLPFC of the left hemisphere. Optode spacing was $45 \mathrm{~mm}$ corresponding to a depth of $20-25 \mathrm{~mm}$. Following instructions by Holtzer and colleagues [14], changes in oxygenated $\left(\mathrm{O}_{2} \mathrm{Hb}\right)$ and deoxygenated $(\mathrm{HHb})$ hemoglobin concentrations relative to a 5-s baseline, recorded immediately before the first condition, were calculated for each condition. More specifically, for a given $60 \mathrm{~s}$ trial the first and last $10 \%$ of the fNIRS data were deleted, meaning each trial had continuous data for $48 \mathrm{~s}$ in duration. This was to reduce measurement error at the beginning and end of each trial.

\section{Statistical analysis}

For all parameters, the average of the two trials of each condition was used for further analysis. Data were analyzed with IBM SPSS Statistics 24.0 software for Windows (SPSS, Inc., Chicago, Ill, USA). Homogeneity of variances and normality of the distribution of the parameters was tested with the Levene's and Shapiro-Wilk's test, respectively. Two-way mixed-design analyses of variance (ANOVA) with age as a between-subject factor (young vs. older adults) and condition as a within-subject factor (ST vs. DT) was performed on the COP parameters as well as on the number of correct answers for the cognitive task. The hemodynamic variables $\left(\mathrm{O}_{2} \mathrm{Hb}\right.$ and $\left.\mathrm{HHb}\right)$ were also analyzed using a two-way mixed design ANOVA with the within- and between-subjects factors of age (young vs. older adults) and condition (four levels: Baseline, Cognitive ST, Tandem ST, DT). Significant main or interaction effects were followed up by Bonferroni-corrected post hoc tests. Finally, for the subjective ratings of physical and cognitive load, a Friedman's ANOVA was used. Statistical significance was set at the level of $p<0.05$.

\section{Results \\ Baseline characteristics}

Table 1 summarizes the participants' characteristics. The independent sample $t$-test showed that participants varied by age $(p<0.001)$ but were well-matched for all other parameters $(p \geq 0.074)$. Furthermore, older adults showed no signs of cognitive impairments (MoCA score $\geq 26$ ).

\section{Postural control}

Analysis showed no significant main or interaction effects for parameters of COP sway path (total, m-l, and a-p; all $p$ s $>0.141)$.

\section{Cognitive performance}

A significant interaction effect of age and condition (Cognitive ST vs. DT), $F(1,18)=10.655, p=0.004$, partial $\eta^{2}=$ 0.372 , was found for the number of correct answers. Young 
adults gave more correct answers than older adults during the ST $(p=0.027)$ as well as during the DT $(p=0.003)$ condition. Moreover, young adults increased the number of correct answers from the ST to the DT condition $(p=$ 0.002 ), whereas there was no difference between conditions in older adults (see Fig. 1).

\section{Cognitive-motor dual-task interference}

In the cognitive-motor dual-task interference analysis, older adults show a mutual interference, while young adults show mutual facilitation where the majority of improvements were found in cognitive task and almost no change $(+0.3 \%)$ in COP parameters (see Fig. $1 \mathrm{c})$. Here the only significant change remains the cognitive improvements in young adults from Cognitive ST to DT $(p=0.002)$, and there are no changes observed in the older adults $(p=0.404)$. In the change of COP parameters from Tandem ST to DT, no differences were observed in younger $(p=0.756)$, nor older participants $(p=0.112)$. Moreover, also in the oxygenated hemoglobin no changes from Tandem ST to DT in young $(p=0.597)$, nor old $(p=0.752)$.

\section{Hemodynamic changes}

Hemodynamic changes for $\mathrm{O}_{2} \mathrm{Hb}$ values revealed no significant interaction effect $(p=0.898)$, however there was a significant main effect of condition on $\mathrm{O}_{2} \mathrm{Hb}$ values (see Fig. 2a), $F(3,54)=7.329, p=0.002$, partial $\eta^{2}=0.289$. Post hoc tests revealed a significant change from Baseline to Tandem ST $(p=0.033)$, and Baseline to DT $(p=0.031)$, while there were no significant changes from Baseline to Cognitive ST $(p=0.641)$ and Tandem ST to DT $(p=0.430)$. Finally, for $\mathrm{HHb}$ values, there was no significant interaction $(p=0.890)$, nor main effect of condition $(p=0.663)$.

\section{Subjective rating of physical and cognitive load}

Fig. 2 b depicts Borg values separately for younger and older adults, as well as for both groups together. There was a significant difference in the perceived effort between conditions $\left(X^{2}(3)=43.006, p<0.001\right)$. Post hoc tests were significant between all conditions $(p \leq 0.003)$ except between Tandem ST and DT $(Z=-1.271, p=$ 0.204). Additionally, no significant differences were observed between the two age groups for all four conditions for the subjective rating of Borg scale $(p \geq 0.063)$ : no differences were obtained for Baseline $(p=0.676)$, Cognitive ST $(p=0.507)$ and Tandem ST $(p=0.147)$, with a non-significant trend for DT $(p=0.063)$

\section{Discussion}

This study investigated a postural-cognitive dual-task paradigm in young and older adults using fNIRS. The study revealed significant increases in $\mathrm{O}_{2} \mathrm{Hb}$ in the DLPFC when the difficulty of the postural task was increased (i.e., from normal to tandem stance), whereas there were no changes when cognitive load was added to standing. This was true for both age groups with no significant age differences in oxygenation levels. For the behavioral parameters, no significant differences between age groups or conditions were found except for a slightly better performance in the cognitive task from the single- to the dual-task condition in young adults. Finally, a subjective measure of physical and cognitive load revealed no age-related differences and was found to be significantly different between all conditions expect when cognitive load was added to a tandem postural stance.

There were no significant differences observed in DTEs in either the younger or older adults with an exception of improvements observed in the subtracting task in the young adults. When addressing the motor-cognitive dual-task interference, older adults show a mutual interference (slight cognitive as well as postural interference), while young adults show a mutual facilitation (slight postural facilitation and a significant cognitive facilitation) during the most demanding tandem stance condition. The latter could be indicative of higher concentration levels and/or attentional resources recalled during the more demanding postural condition. Accordingly, no differences in prefrontal oxygenation was found between the single- and the dual-task conditions or between age groups. To the contrary, Rosso et

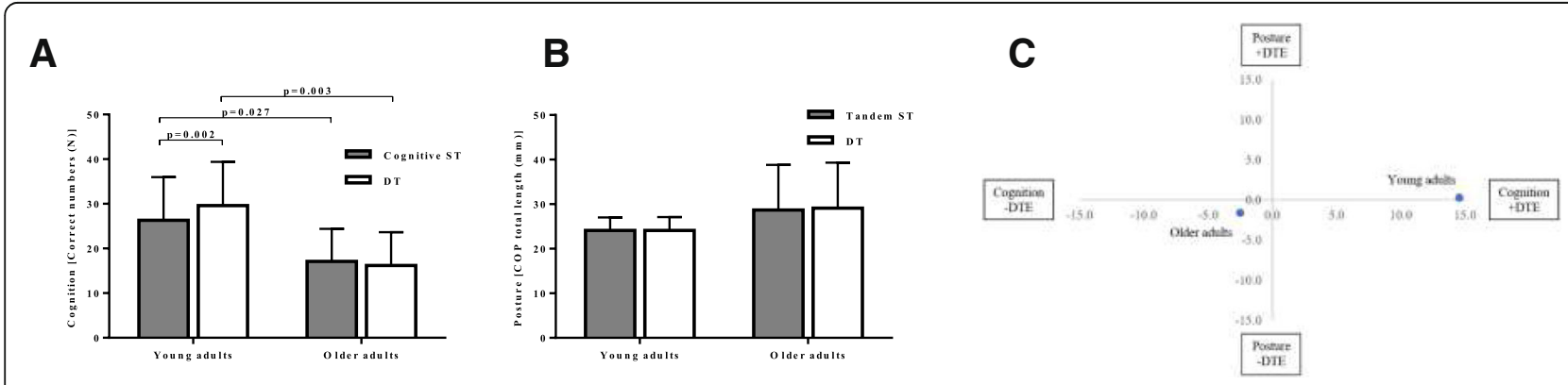

Fig. 1 Cognitive performance (serial threes) during Cognitive ST and DT positions (a); Total length of body sway in millimeters (b); Cognitive-motor dual-task interference $(\mathbf{c})$ 

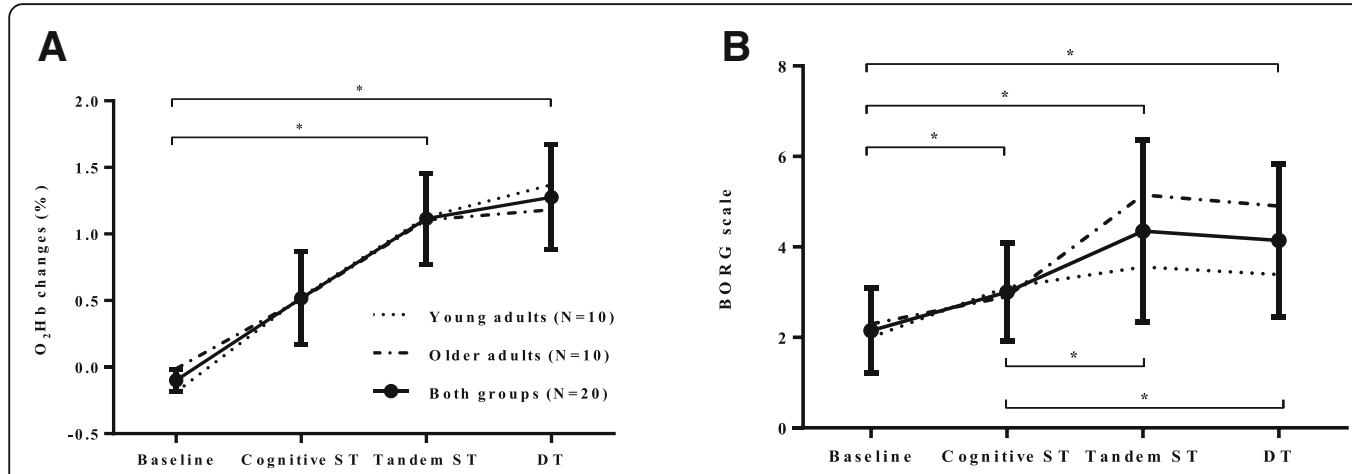

Fig. $2 \mathrm{O} 2 \mathrm{Hb}$ changes in all four conditions (a); BORG scale of perceived exertion (b) Note: no significant differences were observed between the two groups. However, significant changes between conditions are marked with *. The calculation of relative $\mathrm{O}_{2} \mathrm{Hb}$ is stated in paragraph "2.3 Cerebrovascular setup".a.

al. [27] reported higher activation patterns of the prefrontal cortex and temporal regions in older, compared to young adults. Our results are not directly comparable with theirs due to the fact that they used a different cognitive task (auditory choice reaction time) as well as a different postural task (dynamic stance with feet together and eyes closed). Similar findings than ours were reported in a study that compared walking alone to walking while talking [23]. Other studies using similar paradigms, however, found increased oxygenation levels in the prefrontal cortex during walking while talking compared to normal walking in both young and older adults $[14,15]$. The discrepancy between these findings and our results could be explained by differences in the attentional demands of the postural tasks used; possibly, the two-legged standing tasks used in the present study were not challenging enough to induce significant effects. It has been shown in a systematic review [5] that static standing tasks are not always challenging enough to detect age-related changes in dual-task abilities. Indeed, studies using postural tasks with dynamic surface conditions systematically reported greater dual-task costs in older compared to young adults [5]. Similarly, it has been shown that walking is more attention-demanding than standing, in both young and older adults, possibly due to the particular demands of generating bilateral, synchronized limb movements [28]. Further support for this interpretation comes from a study that combined standing tasks with a cognitive task and found increases in DLPFC oxygenation during dual-task only in a one-legged standing condition and not during two-legged standing [29].

Interestingly, one study reported a smaller increase in prefrontal activity from single- to dual-task walking in older compared to young adults [14] while another study even found a deactivation in older adults in one dual-task condition while in young adults there was no change [23]. This might be somewhat surprising since it has been shown that performing postural and cognitive tasks alone increases prefrontal activity more in older than in young adults [3]. It has been suggested that older adults may underutilize the prefrontal cortex when performing an attention-demanding task while walking [14]. This suggestion is supported by two studies [30,31] showing that transcranial direct current stimulation of the prefrontal cortex improves dual-task performance in older adults. In these studies, the costs of performing a working memory task concurrently with standing or walking were significantly reduced when prefrontal activity was facilitated by the stimulation while it had no influence on single-task performances. Another interpretation is that older adults shift processing resources to other brain areas in dual-task situations [23]. Since in the present study, brain activity was measured only within a limited area of the prefrontal cortex, namely DLPFC, we cannot exclude activity changes in other brain areas in the older adults.

There are some methodological limitations that should be taken into consideration when interpreting the present study. Firstly, the fNIRS system allowed us to perform measurements only within a limited area of the prefrontal cortex, namely DLPFC. Future studies could include a multichannel fNIRS device to cover a larger as well as bilateral brain regions, as similar studies have revealed postureand age-related interactions in other brain areas, such as the sensorimotor cortex (e.g., Wang et al. [32]). Moreover, a prefrontal hemispheric asymmetry can be found after different cognitive-motor interventions (e.g. an elevation of left ventrolateral PFC activity and a reduction of right ventrolateral PFC activity has been observed in old adults after cognitive training [33] or cognitive-motor training [34]. Secondly, due to the fact that normal aging is also accompanied by declines in executive function, including deficits in processing speed, working memory capacity, and attentional processing (namely in tasks requiring selective and/or divided attention) [35], other cognitive loads (e.g., reciting alternate letters of the alphabet, mental rotation task, serial sevens) aside from serial threes need to be 
tested. Another limitation goes in the direction of greater differences in age-related discrimination, that would perhaps have been more visible if the variability of COP parameters were available (e.g., standard deviations and/or root mean squares; for useful review see [36]). We further acknowledge that our sample of healthy older adults might not be representative of the general population of older adults. This assumption is supported by the older participants' subjective ratings of their health and fitness status (see Table 1), which were above the average among other older adults we collected in a larger PANGeA cohort (not published observations). Finally, although small sample sizes between groups are common in the literature, it must be acknowledged here that the matched $n=10$ samples for each age group represent only the minimum needed for conducting the statistical parameters of the present pilot study (typically between 8 and 10 individuals per homogeneous group).

\section{Conclusions}

In summary, this is the first study to provide an indication that increasing postural demands contributes more to the observed increase in DLPFC activity during a postural dual-task than increasing the cognitive load. However, it should be noted that this finding may also have arisen from the fact that an increase in cognitive demand (i.e., simple mental math: "counting backwards by threes") may not be directly comparable to the increase in motor demand (i.e., narrowing the base of support: "tandem stance"). The fNIRS measurements showed significantly higher $\mathrm{O}_{2} \mathrm{Hb}$ concentration level changes in the DLPFC when postural task difficulty was increased (from baseline to Tandem ST). In contrast, there were no significant increases in $\mathrm{O}_{2} \mathrm{Hb}$ concentration between the baseline and the dual-task condition (Cognitive ST) nor tandem stance (Tandem ST) and the DT condition. This suggests that changing the posture from normal stance to tandem stance increases cognitive load, at least in the DLPFC, while adding a serial threes cognitive task to tandem stance does not. Understanding the neural contribution to reduced postural control in aging could help developing novel interventions for elderly to reinforce postural control, especially in challenging dual- and multi-task conditions, and improve their quality of life, including a faster return to premorbid daily functioning.

\section{Acknowledgements}

The authors would like to thank the participants in the study, and all members of the research team. Additionally, we thank Kaja Teraz for randomization of subjects, and her help with data collection.

\section{Funding}

Slovenian Research Agency, P5-0381.

\section{Authors' contributions}

UM corresponding author, concept creation and design, measurements, data analysis, interpretation of data, drafted manuscript, WT and SM concept creation, interpretation of data, critically reading and proof-reading the manuscript, LB and BG concept creation, interpretation of data, KP, RM and $\mathrm{RP}$ assistance in the design of the article, proof-reading the manuscript, JR assistance in writing and in designing, critically reading and proof-reading the manuscript. All authors read and approved the final manuscript.

\section{Ethics approval and consent to participate}

The present study was part of a larger EU project PANGeA and approved by the National Medical Ethics Committee no. 131/05/14. Written informed consent was obtained from all participants prior to the study and no payment was provided for participation.

\section{Consent for publication}

Not applicable - but consent was obtained from all included subjects.

\section{Competing interests}

The authors declare that they have no competing interests.

\section{Publisher's Note}

Springer Nature remains neutral with regard to jurisdictional claims in published maps and institutional affiliations.

\section{Author details \\ ${ }^{1}$ Institute for Kinesiology Research, Science and Research Centre Koper, Garibaldijeva 1, Koper, Slovenia. ${ }^{2}$ Department of Health Sciences, Alma Mater Europaea - ECM, Maribor, Slovenia. ${ }^{3}$ Department of Neurosciences and Movement Sciences, University of Fribourg, Fribourg, Switzerland. ${ }^{4}$ Department of kinesiology and physiotherapy, Faculty of Health Sciences, University of Primorska, Izola, Slovenia. ${ }^{5}$ Department of Medical and Biological Sciences, Udine University, Udine, Italy. ${ }^{6}$ Research Group Human Physiology, Vrije Universiteit Brussel, Brussels, Belgium. ${ }^{7}$ Faculty of sport, University of Ljubljana, Ljubljana, Slovenia.}

Received: 17 October 2018 Accepted: 27 December 2018 Published online: 11 January 2019

\section{References}

1. Papegaaij S, Taube W, Baudry S, Otten E, Hortobagyi T. Aging causes a reorganization of cortical and spinal control of posture. Front Aging Neurosci. 2014;6:28.

2. Zwergal A, Linn J, Xiong G, Brandt T, Strupp M, Jahn K. Aging of human supraspinal locomotor and postural control in fMRI. Neurobiol Aging. 2012; 33(6):1073-84

3. Seidler RD, Bernard JA, Burutolu TB, Fling BW, Gordon MT, Gwin JT, et al. Motor control and aging: links to age-related brain structural, functional, and biochemical effects. Neurosci Biobehav Rev. 2010;34(5):721-33.

4. Holtzer R, Epstein N, Mahoney JR, Izzetoglu M, Blumen HM. Neuroimaging of mobility in aging: a targeted review. J Gerontol A Biol Sci Med Sci. 2014; 69(11):1375-88.

5. Boisgontier MP, Beets IA, Duysens J, Nieuwboer A, Krampe RT, Swinnen SP. Age-related differences in attentional cost associated with postural dual tasks: increased recruitment of generic cognitive resources in older adults. Neurosci Biobehav Rev. 2013;37(8):1824-37.

6. Ruffieux J, Keller M, Lauber B, Taube W. Changes of standing and walking performance under dual-task conditions across the lifespan: a systematic review. Sports Med. 2015;45(12):1739-58.

7. Little CE, Woollacott M. Effect of attentional interference on balance recovery in older adults. Exp Brain Res. 2014;232(7):2049-60.

8. Tsang WW, Lam NK, Lau KN, Leung HC, Tsang CM, Lu X. The effects of aging on postural control and selective attention when stepping down while performing a concurrent auditory response task. Eur J Appl Physiol. 2013;113(12):3021-6.

9. Lindenberger U, Marsiske M, Baltes PB. Memorizing while walking: increase in dual-task costs from young adulthood to old age. Psychol Aging. 2000; 15(3):417-36.

10. Krampe RT, Schaefer S, Lindenberger U, Baltes PB. Lifespan changes in multi-tasking: concurrent walking and memory search in children, young, and older adults. Gait Posture. 2011;33(3):401-5. 
11. Chen HC, Schultz AB, Ashton-Miller JA, Giordani B, Alexander NB, Guire KE. Stepping over obstacles: dividing attention impairs performance of old more than young adults. J Gerontol A Biol Sci Med Sci. 1996;51(3):M116-22.

12. Zijlstra A, Ufkes T, Skelton DA, Lundin-Olsson L, Zijlstra W. Do dual tasks have an added value over single tasks for balance assessment in fall prevention programs? A mini-review Gerontology. 2008;54(1):40-9.

13. Beauchet O, Annweiler C, Dubost V, Allali G, Kressig RW, Bridenbaugh S, et al. Stops walking when talking: a predictor of falls in older adults? Eur J Neurol. 2009;16(7):786-95.

14. Holtzer R, Mahoney JR, Izzetoglu M, Izzetoglu K, Onaral B, Verghese J. fNIRS study of walking and walking while talking in young and old individuals. J Gerontol A Biol Sci Med Sci. 2011;66(8):879-87.

15. Holtzer R, Mahoney JR, Izzetoglu M, Wang C, England S, Verghese J. Online fronto-cortical control of simple and attention-demanding locomotion in humans. Neurolmage. 2015;112:152-9.

16. Mihara M, Miyai I, Hatakenaka M, Kubota K, Sakoda S. Role of the prefrontal cortex in human balance control. Neurolmage. 2008;43(2):329-36.

17. Basso Moro S, Bisconti S, Muthalib M, Spezialetti M, Cutini S, Ferrari M, et al. A semi-immersive virtual reality incremental swing balance task activates prefrontal cortex: a functional near-infrared spectroscopy study. Neurolmage. 2014;85(Pt 1):451-60.

18. Ferrari M, Bisconti S, Spezialetti M, Basso Moro S, Di Palo C, Placidi G, et al. Prefrontal cortex activated bilaterally by a tilt board balance task: a functional near-infrared spectroscopy study in a semi-immersive virtual reality environment. Brain Topogr. 2014;27(3):353-65.

19. Mahoney JR, Holtzer R, Izzetoglu M, Zemon V, Verghese J, Allali G. The role of prefrontal cortex during postural control in parkinsonian syndromes a functional near-infrared spectroscopy study. Brain Res. 1633;2016:126-38.

20. Petrides $M$. The role of the mid-dorsolateral prefrontal cortex in working memory. Exp Brain Res. 2000;133(1):44-54.

21. McCarthy G, Blamire AM, Puce A, Nobre AC, Bloch G, Hyder F, et al. Functional magnetic resonance imaging of human prefrontal cortex activation during a spatial working memory task. Proc Natl Acad Sci U S A. 1994;91(18):8690-4.

22. Barbey AK, Koenigs M, Grafman J. Dorsolateral prefrontal contributions to human working memory. Cortex. 2013;49(5):1195-205.

23. Beurskens R, Helmich I, Rein R, Bock O. Age-related changes in prefrontal activity during walking in dual-task situations: a fNIRS study. Int J Psychophysiol. 2014;92(3):122-8.

24. Marusic U, Kavcic V, Giordani B, Gerzevic M, Meeusen R, Pisot R. Computerized spatial navigation training during 14 days of bed rest in healthy older adult men: effect on gait performance. Psychol Aging. 2015

25. Plummer P, Eskes G. Measuring treatment effects on dual-task performance: a framework for research and clinical practice. Front Hum Neurosci. 2015;9:225.

26. Salvadego D, Lazzer S, Marzorati M, Porcelli S, Rejc E, Simunic B, et al. Functional impairment of skeletal muscle oxidative metabolism during knee extension exercise after bed rest. J Appl Physiol (1985). 2011;111(6):1719-26.

27. Rosso AL, Cenciarini M, Sparto PJ, Loughlin PJ, Furman JM, Huppert TJ. Neuroimaging of an attention demanding dual-task during dynamic postural control. Gait and posture. 2017;57:193-8.

28. Yogev-Seligmann G, Giladi N, Gruendlinger L, Hausdorff JM. The contribution of postural control and bilateral coordination to the impact of dual tasking on gait. Exp Brain Res. 2013;226(1):81-93.

29. Fujita H, Kasubuchi K, Wakata S, Hiyamizu M, Morioka S. Role of the frontal cortex in standing postural sway tasks while dual-tasking: a functional nearinfrared spectroscopy study examining working memory capacity. Biomed Res Int. 2016;2016:10

30. Manor B, Zhou J, Jor'dan A, Zhang J, Fang J, Pascual-Leone A. Reduction of dual-task costs by noninvasive modulation of prefrontal activity in healthy elders. J Cogn Neurosci. 2016;28(2):275-81.

31. Zhou D, Zhou J, Chen H, Manor B, Lin J, Zhang J. Effects of transcranial direct current stimulation (tDCS) on multiscale complexity of dual-task postural control in older adults. Exp Brain Res. 2015;233(8):2401-9.

32. Wang B, Zhang M, Bu L, Xu L, Wang W, Li Z. Posture-related changes in brain functional connectivity as assessed by wavelet phase coherence of NIRS signals in elderly subjects. Behav Brain Res. 2016;312:238-45.

33. Erickson Kl, Colcombe SJ, Wadhwa R, Bherer L, Peterson MS, Scalf PE, et al. Training-induced plasticity in older adults: effects of training on hemispheric asymmetry. Neurobiol Aging. 2007;28(2):272-83.
34. Eggenberger $P$, Wolf $M$, Schumann $M$, de Bruin ED. Exergame and balance training modulate prefrontal brain activity during walking and enhance executive function in older adults. Front Aging Neurosci. 2016;8:66.

35. Harada CN, Natelson Love MC, Triebel KL. Normal cognitive aging. Clin Geriatr Med. 2013;29(4):737-52.

36. Paillard T, Noé F. Techniques and methods for testing the postural function in healthy and pathological subjects. Biomed Res Int. 2015;2015.

\section{Ready to submit your research? Choose BMC and benefit from:}

- fast, convenient online submission

- thorough peer review by experienced researchers in your field

- rapid publication on acceptance

- support for research data, including large and complex data types

- gold Open Access which fosters wider collaboration and increased citations

- maximum visibility for your research: over $100 \mathrm{M}$ website views per year

At BMC, research is always in progress.

Learn more biomedcentral.com/submissions 